\title{
The Research on Energy Releasing Control of Travelling Charge
}

\section{LIANG Tai-xin ${ }^{1,}$, LV Bing-feng ${ }^{2}$, MA Zhong-liang ${ }^{3}$, XIAO Zhong-liang ${ }^{4}$}

${ }^{1}$ School of Chemical Engineering and Environment, North University of China, Taiyuan, China

aliangtx2006@126.com

Keywords: ordnance science and technology; traveling charge; combustion performance; delayed ignition; gun propellant

\begin{abstract}
For solving timely ignition of travelling charge and regular energy releasing, a new mode of travelling charge has been proposed. Propellant is substrate propellant, adopt existing energetic binder, use the energetic binder with lower burning rate and energy by compaction technology to shrink and wrap the propellant with higher burning rate and energy, press it into travelling charge cylindrical grain with high density and high strength, and achieve the purpose of controlling energy releasing control of travelling charge. Clad the end of the cylindrical grain with newly developed slow-burning material, combine cylindrical grain's compactness, and achieve the timely ignition of travelling charge. And research it through closed-vessel experiments, the results show that combine the cylindrical grain's compactness, travelling charge timely ignition can achieve by slow-burning layer; the whole combustion performance of travelling charge is stable, repeatability is good, it has good macro properties of progressive combustion and energy release rule.
\end{abstract}

\section{Introduction}

Travelling charge is a charge technology that can make energy compensation to gun bore, delay chamber pressure's quick drop because of projectile's high speed when reach to the maximum chamber pressure, increasing the pressure area under the curve to reach to similar pressure platform effect, can meet the necessary and sufficient condition of improving muzzle energy $[1,3]$. Domestic scholars have conducted relevant research towards energy releasing control of travelling charge. Michel et al. [4] apply the steady state deflagration technology to travelling charge, make a shoot experiment at 30mm gun,projectile initial velocity have increased $40 \%-60 \%$, but they can't control energy release efficiently and the chamber pressure increase a lot, and haven't achieved breakthrough yet. Stobie et al. [5] make a experiment to liquid propellant travelling charge by piston-separating method, the result shows that the experiment's repeatability is poor if don't control travelling charge combustion and the time delay ignition system is not stable. Dong Y L et al. [6] make flame retardant-coated propellant as travelling charge and make an experiment at $105 \mathrm{~mm}$ gun, muzzle initial velocity has respectively increased $2.8 \%$ and $4.2 \%$ at $330 \mathrm{MPa}$ and $400 \mathrm{MPa}$, although it has some control towards travelling charge energy, but the effect is not ideal, initial velocity doesn't up to the expectation. Xin L, Hua Z et al. [7,8] apply movement differential principle to realize the continuous injection of liquid propellant toward the in-bore space and the control of liquid propellant travelling charge energy release, when the chamber pressure maximum is invariant, the initial velocity increased $94 \mathrm{~m} / \mathrm{s}$ than conventional charge. So, it has become an urgent issue to solve travelling charge timely ignition and regular energy release.

This study is on the basis of travelling charge propellant physical structure invariance, blends it with binder at appropriate scale, models it under certain pressure, prepare a travelling charge cylindrical grain with high density and high strength, achieve regular energy release towards travelling charge while further improve loading energy. Adopt end cladding towards travelling charge cylindrical grain by a slow-burning material, combine cylindrical grain's compactness, achieve travelling charge's timely ignition. 


\section{Technical Way and experimental}

Technical Way. Tubular propellant $2 / 1$ propellant as the substrate propellant, premix it with 5\% energetic binder, put it in kneading mixer and knead 30min so that energetic binder can fully infiltrate towards substrate propellant's surface, substrate propellant can fully agglomerate, then use compression moulding in tailor-made steel cylinder at $4 \mathrm{MPa}$, the pressed density is $1.55 \mathrm{~g} / \mathrm{cm}^{3}$. The tailor-made steel cylinder can simulate travelling charge combustion in closed-vessel experiments, substrate propellant and energetic binder are distributed evenly after shaped charge. The energetic binder is made of proportional double cobalt propellant and TEGN and 2\#NC [9].

Slow-burning material's major ingredient is low energy gunpowder with low burning rate, and adds a certain amount of EC. The merits are: the materials used are combustible, the chemical reaction has no residual; $1 \mathrm{~g}$ dosage can has ignition delay effect, the negative quality produced is less; it can bond effectively with cylindrical grain end with good viscosity, small springiness, high plasticity; the chemical property is stable, hard to deform, it can be stored for a long time.

After end cladding towards travelling charge cylindrical grain, the overall structure is shows in Fig. 1.
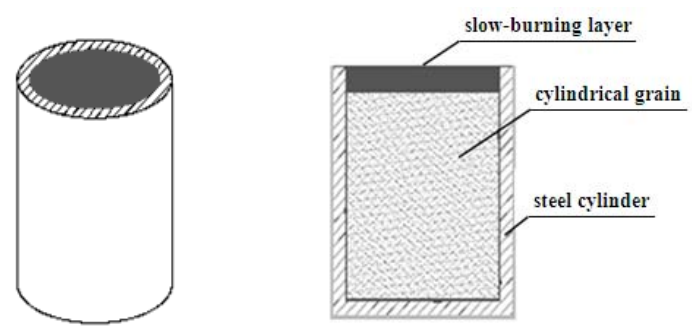

(a) Free-space diagram (b) Cross-section drawn

Fig.1 Schematic drawing traveling charge structure

Experimental. In order to simulate the combustion environment of travelling charge in chamber, propellant $6 / 7$ is the main charge and makes a closed-vessel experiment with travelling charge sample by mixed charge. The experimental condition is: closed-vessel volume is $200 \mathrm{~cm}^{3}$; loading density is $0.2 \mathrm{~g} / \mathrm{cm}^{3}$; experimental temperature is $20^{\circ} \mathrm{C}$; ignition powder is $2 \# \mathrm{NC}$; ignition pressure is $10 \mathrm{MPa}$; pressure transducer measurement range is $0 \sim 900 \mathrm{MPa}$; pressure transducer precision $0.005 \mathrm{MPa}$; sample interval is $0.05 \mathrm{~ms}$; relative humidity is not more than $75 \%$.

\section{Results and Discussion}

Fig. 2 is the contrast of $40 \mathrm{~g}$ propellant closed-vessel curve and $36 \mathrm{~g} \mathrm{6/7}$ propellant mixed with travelling charge sample closed-vessel curve, curve 2, 3 have same charge condition. The content of travelling charge is $11 \mathrm{~g}$; slow-burning layer's thickness is $0.8 \mathrm{~mm}$.

The figure shows that three curves coincide from the burning start to $53.8 \mathrm{~ms}$, then curve 2,3 are higher than curve 1, pressure increase rapidly to the maximum. This shows that combustion in closed-vessel by mixed charge has two stages: first stage is the common combustion of main charge $6 / 7$ and travelling charge sample' slow-burning layer. The second stage is the common combustion of $6 / 7$ propellant and travelling charge cylindrical grain. Corresponded with curve 2 , the first stage is from the burning start to $53.8 \mathrm{~ms}$, this stage's pressure increase depends on $6 / 7$ propellant's combustion. Slow-burning layer burns out at $53.8 \mathrm{~ms}$, and enter into second stage-the common combustion of $6 / 7$ propellant and travelling charge grain, the two propellants' combustion resolve this stage's pressure increase.

It also shows that the existence of two stages is due to travelling charge slow-burning layer's slow-burning effect. This indicate that slow-burning layer can isolate travelling charge cylindrical grain and high temperature flame in a given time frame, can control effectively towards travelling charge grain's ignition time at high temperature and high pressure. The relative experiments show that travelling charge grain's ignition delay with the increase of slow-burning layer thickness when the content of travelling charge cylindrical grain energetic binder is $2 \% \sim 9 \%$, the pressed density is 
$(1.5 \sim 1.6) \mathrm{g} / \mathrm{cm}^{3}[10]$

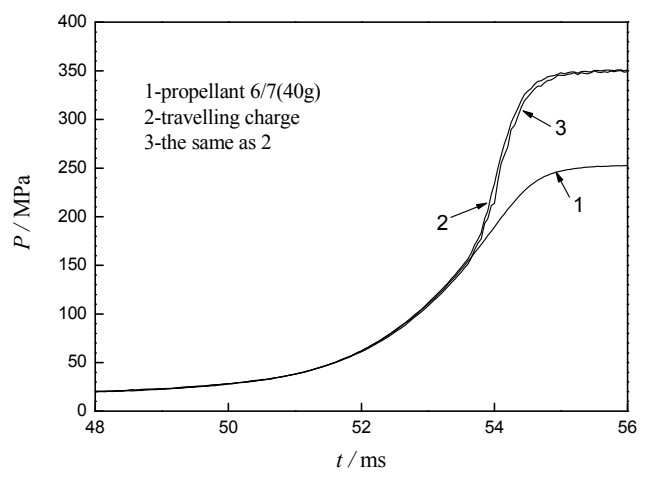

Fig. 2 p-t curves of samples

Curve 2, 3 in fig. 2 show that the two curves have better contact ratio from combustion start to maximum pressure spot. It indicates that this travelling charge energy release regularity overlapping consensus is better in same charge condition; travelling charge slow-burning layer has better overlapping consensus towards grain ignition time; the combustion performance of travelling charge cylindrical grain is stable, overlapping consensus is better.

Fig. 3 shows relative $L-B$ curve, it indicates that dynamic vivacity of travelling charge is lower than $6 / 7$ propellant when relative pressure is $[0.1,0.38]$, dynamic vivacity increases rapidly when relative pressure is 0.38 , dynamic vivacity is above $6 / 7$ propellant at 0.45 and 0.9 . It indicates that travelling charge start combusting when relative pressure is 0.38 , the whole rate of gas production in gun bore increases, travelling charge has good macroscopic properties of progressive combustion. Meanwhile, the curve is smoother which indicates travelling charge has better energy release regularity.

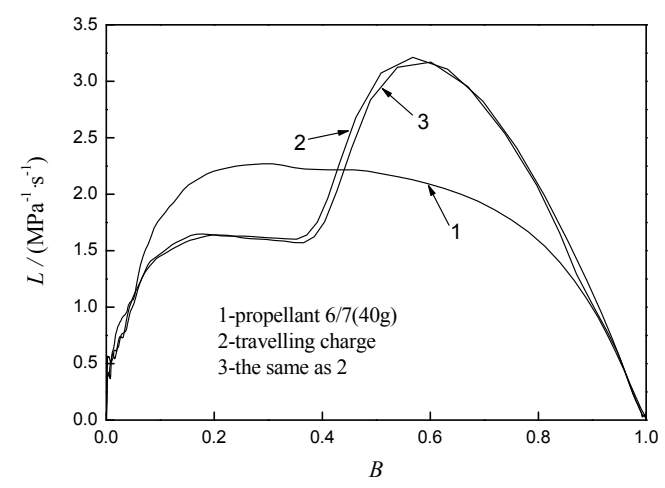

Fig. $3 L-B$ curves of samples

As to travelling charge cylindrical grain, its combustion has properties of progressive combustion as well [11]. At the combustion start stage, the pressure of effective suppressing propellant combustion's start stage increases due to the whole compactness of grain and smaller burning area. With energetic binder's combustion, the cylindrical grain disintegrate into propellant grains, the combustion of propellant's surface is at energetic binder membrane's defects. With energetic binder's complete combustion, burning area and burning rate increase which shows properties of progressive combustion. So, the mixture compaction of energetic binder and propellant, and travelling charge method of end cladding by slow-burning material can control effectively towards travelling charge energy release.

\section{Summary}

This article researches new travelling charge through closed-vessel experiments. We can draw the following conclusions by analyzing the experiment result. Combine the cylindrical grain's 
compactness, travelling charge timely ignition can achieve by slow-burning layer; the whole combustion performance of travelling charge is stable, repeatability is good, it has good macro properties of progressive combustion and energy release rule.

\section{References}

[1] Xiao Zhong-liang. The analysis of the technical way and potentiality on improving initial velocity of gun[J]. Journal of North China Institute of Technology, 2001, 22(4): 277 280.

[2] Wang Qiong-lin, Liu Shao-wu, Zhang Yuan-bo, et al. Conception and principle of controlled burning gun propellant[J]. Chinese Journal of Explosives and Propellants, 2009, 32(5): 71 74.

[3] Wang Ze-shan, He Wei-dong, Xu Fu-ming. Propellant charge design principle and technology[M]. Beijing: Beijing institute of technology press, 2006: 250 258.

[4] Michel S R. Application of stationary deflagration to traveling charges. 14th International Symposium on Ballistics, 1993.

[5] Stobie I C, Knapton J D. Test results from a two-stage traveling charge liquid propellant gun. AD-A 224593, 1990.

[6] Dong Y L, Zhen Y Z, Yong G Y, et al. Experiments on the combustion characteristics of deterrent-coated propellants and their application in traveling charge propulsion[J]. Combust Science and Technology, 2012, 184: 178 185.

[7] Xin L, Yan H Z, Yong G Y. Experimental and numerical investigations on traveling charge gun using liquid fuels[J]. Journal of Applied Mechanics, 2011, 78(5): 567 574.

[8] Zou Hua, Lu Xin, Zhou Yan-huang. Theoretical study on interior ballistics of high velocity gun based on liquid traveling charge[J]. Journal of Ballistics, 2013, 25(3): 75 78.

[9] Ma Zhong-liang, Xia Ping, He Zeng-di, et al. Combustion properties of a glued consolidation gun propellant[J]. Chinese Journal of Explosives and Propellants, 2006, 29(5): 60 62.

[10] Fan Xue-kun. The study of energy releasing control based on grain binding propellants[D]. Master Dissertation. Taiyuan: North University of China, 2013.

[11] Yao Yue-juan, Liu Shao-wu, Wang Qiong-lin, et al. Burning performance of grain-molded propellant [J]. Chinese Journal of Energetic Materials, 2012, 20(1): 76 79. 